\title{
Oncogenic viruses and cancer
}

\author{
Guangxiang George Luo ${ }^{1,2 \bowtie}$, Jing-hsiung James $\mathrm{Ou}^{3 凶}$
}

\section{Department of Microbiology, University of Alabama at Birmingham School of Medicine, Birmingham 35294, USA \\ 2. Peking University Health Science Center, Beijing 100083, China \\ 3. Department of Molecular Microbiology and Immunology, University of Southern California Keck School of Medicine, Los Angeles 90033, USA}

This special issue of the journal is dedicated to the important topic of oncogenic viruses and cancer. It contains seven review articles covering all known oncogenic viruses except for human T-lymphotropic virus type 1 (HTLV-1). These review articles are contributed by experts on specific viruses and their associated human cancers.

Viruses account for about $20 \%$ of total human cancer cases. Although many viruses can cause various tumors in animals, only seven of them are associated with human cancers and are currently considered oncogenic viruses. These viruses include hepatitis B virus (HBV), hepatitis C virus (HCV), human papillomavirus (HPV), Epstein Barr virus (EBV), human herpes virus 8 (HHV8), Merkel cell polyomavirus (MCPyV), and HTLV-1. HBV and HCV cause approximately $80 \%$ of hepatocellular carcinoma (HCC), the most common cancer of the liver. High-risk HPV strains are the major causes of cervical cancer and other ano-genital neoplasms as well as a significant proportion of head and neck tumors. EBV is associated with nasopharyngeal carcinoma, Hodgkin's lymphoma, and Burkitt's lymphoma. HHV8 (also called Kaposi's sarcoma-associated herpesvirus, KSHV) is responsible for Kaposi's sarcoma often found in patients with acquired immunodeficiency syndrome (AIDS). MCPyV causes Merkel cell carcinoma and HTLV-1 is the causative agent of adult T-cell lymphoma.

The molecular mechanisms of viral oncogenesis are complex and may involve the induction of chronic inflammation, disruption of host genetic and epigenetic integrity and homeostasis, and interference with cellular DNA repair mechanisms resulting in genome instability and cell cycle dysregulation. Oncogenic DNA viruses can also insert their genomic DNA into cellular chromosomes, resulting in genetic abnormity. Viral 'oncoproteins' can activate cellular signaling pathways, alter the expression of cellular genes and microRNAs either transcriptionally or post-transcriptionally, and destabilize or inactivate tumor suppressor proteins and proteins that regulate cell polarity, signal transduction, immune response, and apoptosis. Genetic and epigenetic alterations induced by infection and replication of oncogenic viruses may lead to the appearance and proliferation of cancer stem cells, which are important for the initiation, progression, metastasis, relapse, and chemotherapy resistance of cancers. The importance and underlying molecular mechanisms of specific cellular genes and signaling pathways in viral oncogenesis are subjects of intense research efforts. A great deal of new knowledge on viral oncogenesis has been obtained through the studies of virus-induced tumorigenicity in xenograft animal models. More significantly, a number of new drugs and vaccines have been developed for treatment and prevention of virus-induced cancers.

The authors of each article in this special issue have strived to provide an overview on the state-of-the-art and latest advances in the study of individual oncogenic viruses. Tian and $\mathrm{Ou}$ discuss how genetic and epigenetic alterations induced by HBV contribute to the development of HCC. The hallmark of HCV infection is chronicity, resulting in cirrhosis and HCC. The article by Gale and colleagues focuses on the role of pathogen recognition receptors in the induction of intracellular immunity against HCV infection. Chow discusses several tissue and cell culture systems being used for the study of HPV infection and replication as well as animal model systems of HPV carcinogenesis. Jin and $\mathrm{Xu}$ summarize the latest progresses in the understanding of miRNAs and stem cells in the development of HPV-associated cancers and the significance of the newly developed HPV vaccines in the prevention of 
HPV infection. Tsang and Tsao focus on the importance of latent EBV infection and individual viral genes and miRNAs in the nasopharyngeal carcinoma pathogenesis. The article by Justice, Verhalen, and Jiang focuses on the DNA damage response induced by polyomavirus and its role in the maintenance of host genomic stability. Avey and colleagues provide an update on the importance of epigenetics in the HHV8 (KSHV) replication and pathogenesis along with a discussion on the development of cell culture and animal model systems for the study of HHV8 biology and oncogenesis. These articles focus on the most significant developments in the areas of specific viruses without laboring readers with extraneous details related to viral replication and oncogenesis. Those interested readers are advised to follow references in individual articles for detailed information.

\section{FOOTNOTES}

We would like to thank all authors for their excellent contributions to this special issue. G.X. Luo is supported by Nature Science Foundation of China (NSFC
81130082) and NIH grants AI097318 and AI091953. J.-h. Ou is supported by NIH grants DK094652, DK100257 and CA177337.

$\triangle$ Correspondence:

Guangxiang George Luo

Phone: +1-205-975-2936,

Email: gluo@uab.edu

ORCID: 0000-0002-0354-6020

Jing-hsiung James Ou

Phone: +1-323-442-1720,

Email: jamesou@hsc.usc.edu

ORCID: 0000-0002-8274-5705

Published online: 23 April 2015 